\title{
Examen des méthodes visant à prévenir et à réduire le risque de contracter la maladie de Lyme
}

\author{
Lindsay $\mathrm{LR}^{1^{*}}$, Ogden $\mathrm{NH}^{2}$, Schofield $\mathrm{SW}^{3}$ \\ ${ }^{1}$ Laboratoire national de microbiologie, Agence de la santé publique du Canada, Winnipeg (Manitoba) \\ ${ }^{2}$ Centre des maladies infectieuses d'origine alimentaire, environnementale et zoonotique, Agence de la santé publique du \\ Canada, Saint-Hyacinthe (Québec) \\ ${ }^{3}$ Programme de lutte contre les maladies transmissibles, ministère de la Défense nationale, Ottawa (Ontario)
}

${ }^{*}$ Correspondance : robbin.lindsay@phac-aspc.gc.ca

\section{Résumé}

Contexte : Les cas de la maladie de Lyme et les zones de populations autonomes de tiques vectrices sont à la hausse au Canada. On s'attend à ce que cette tendance se maintienne. Les mesures de prévention à l'égard de la maladie de Lyme s'avéreront donc appropriées pour un nombre croissant de Canadiens.

Objectif : Présenter un résumé des méthodes visant à réduire le risque de morsures de tiques et prévenir la transmission une fois que la tique se nourrit.

Méthodologie : Une recherche documentaire a été menée pour déterminer les méthodes visant à réduire le risque de morsures de tiques et l'abondance de tiques vectrices, ainsi que le risque d'infection par l'agent pathogène de la maladie de Lyme, Borrelia burgdorferi (BB), en cas de morsure d'une tique vectrice.

Résultats : Les approches actuelles de réduction du risque de morsures de tiques ou la prévention des infections par BB une fois que le moustique a mordu, reposent en grande partie sur la personne touchée. Elles comprennent l'utilisation d'insectifuges topiques, l'utilisation de vêtements protecteurs, le fait d'éviter les zones à risque et de retirer les tiques rapidement (idéalement en moins d'un jour) après qu'elles se soient agrippées à la peau. Ces méthodes sont efficaces, mais limitées par leur observance par le sujet. D'autres approches, notamment la modification du terrain ou l'utilisation d'acaricides pour contrôler les tiques, semblent prometteuses dans d'autres pays, mais n'ont pas été largement adoptées au Canada.

Conclusion : La maladie de Lyme continuera de représenter une menace au Canada. En plus des interventions actuelles pour la prévention de piqûres de tiques et la maladie de Lyme, il existe un besoin de nouveaux outils pour aider à réduire le risque de contracter la maladie de Lyme au Canada.

\section{Introduction}

La maladie de Lyme est une grave maladie humaine causée par la bactérie Borrelia burgdorferi (BB). Elle est transmise par certaines espèces de tiques Ixodes : la tique à pattes noires de l'Ouest (Ixodes pacificus) dans certaines régions à l'ouest des Rocheuses, et la tique à pattes noires (Ixodes scapularis) dans certaines régions du Canada, à l'est des Rocheuses. Ces tiques sont infectées lorsqu'elles se nourrissent sur des animaux sauvages hôtes infectés par la bactérie BB, comme des rongeurs ou des oiseaux. Une infectées, elles peuvent transmettre la bactérie BB à d'autres animaux, y compris l'homme (1).

Le risque de contracter la maladie de Lyme au Canada est le plus élevé dans les endroits où les populations de tiques à pattes noires sont établies (p. ex. lorsque les populations sont autonomes d'une année à l'autre) (2). La présence de tiques et le risque varient des deux côtés des Rocheuses. Les populations d'Ixodes pacificus sont largement établies dans le sud de la Colombie-Britannique. Elles ne semblent pas élargir leur portée 
géographique et ont habituellement un faible taux d'infection à la BB $(<5 \%)$. Par conséquent, le risque est relativement modeste. En revanche, l'étendue géographique des populations établies de tiques $I$. scapularis (à l'est des Rocheuses) s'est récemment étendue au centre et à l'est du Canada $(2,3)$ et touche maintenant des zones à proximité des centres urbains ou dans les centres urbains $(4,5)$. En outre, la proportion de tiques I. scapularis infectées par la bactérie BB peut être élevée (> 15\%). L'effet combiné pour certaines régions touchées se solde par un plus grand nombre de tiques infectées se rapprochant des centres de population et entraînant un risque relativement plus élevé de contracter la maladie de Lyme dans le centre et l'est du Canada.

L'incidence annuelle des cas de la maladie de Lyme a nettement augmenté au Canada. Par exemple, en 2004, on recensait seulement 40 cas; toutefois, en 2012, 338 cas étaient signalés (2). Étant donné que l'on s'attend à ce que la tendance à s'étendre du vecteur se maintienne $(2,4)$, la nécessité d'interventions visant à prévenir les morsures de tiques et la maladie de Lyme devient de plus en plus pressante.

Malheureusement, il existe relativement peu de solutions de prévention de la maladie de Lyme disponibles pour les Canadiens. II n'existe aucun vaccin pour les humains (cependant, il existe des traitements efficaces pour la maladie de Lyme); les acaricides pour lutter contre les tiques ne sont pas offerts ou utilisés largement (6); l'expertise et l'infrastructure professionnelles de gestion des tiques sont limitées. Par conséquent, la prévention repose sur les mesures prises individuellement - habituellement par la prévention des piqûres ou par le retrait des tiques sur la peau avant la transmission de la $\mathrm{BB}(7,8,9,10,11)$. L'objectif de cet examen est de résumer les méthodes que le public peut utiliser en vue de prévenir et de prendre en charge les morsures de tiques pour prévenir ou réduire le risque de contracter la maladie de Lyme. L'accent est mis sur des approches visant I. scapularis, mais les recommandations sont généralement applicables à I pacificus et aux autres espèces de tiques.

\section{Méthodologie}

Une recherche documentaire axée sur les mesures utilisées pour prévenir ou contrôler la présence des tiques et leurs morsures a été réalisée. Les bases de données de source ouverte (p. ex. PubMed et le United States Armed Forces Pest Management Board Literature Retrieval System (http://www.afpmb.org/content/welcomeliterature-retrieval-system) ont été consultées pour y trouver des publications pertinentes en utilisant les termes anglais de recherche correspondant au mot français : " tique » et « insectifuge "; " maladie de Lyme » et " prévention » ou « Ixodes scapularis » et « lutte ». Les études, les analyses documentaires ou rapports d'interventions ou de mesures qui se sont démontrées efficaces, qui ont été adoptées dans d'autres pays, et qui avaient une plausibilité biologique de réduire le risque ont été examinées. Les constatations ont été résumées dans un examen narratif et utilisées en vue d'élaborer les lignes directrices et les recommandations suivantes sur la prévention de la maladie de Lyme pour les Canadiens.

\section{Résultats}

Les rapports sur quatre méthodes de prévention ont été résumés : l'évitement des régions à risque; l'utilisation de vêtements protecteurs et la sensibilisation au retrait rapide des tiques; l'utilisation d'une barrière ou d'un insectifuge chimiques; et la réduction des populations de tiques dans l'environnement.

\section{Prévention de la maladie par évitement des régions à risque}

Une règle simple pour lutter contre la maladie de Lyme est : si vous n'êtes pas porteur de tiques, vous ne pouvez pas être malade. Jusqu'à tout récemment, du moins au Canada, cela pouvait se faire en évitant les quelques régions où la maladie de Lyme avait été signalée $(12,13)$. Cependant, en raison de la propagation de I. scapularis et de la maladie de Lyme, les tiques vectrices se trouvent dans de plus nombreuses régions, y compris plus près des centres à forte densité de population, dans ces centres, et même sur les propriétés résidentielles (3). Quoi qu'il en soit, l'évitement demeure une approche de réduction des risques qui a de bonnes chances de réussite (10). 
Les tiques sont associées à certains habitats $(14,15)$, en particulier dans et autour des régions boisées

(Figure 1) qui supportent des populations d'oiseaux, de rongeurs et de chevreuils qui sont les principaux hôtes de la tique à pattes noires (16). Dans ces régions, les tiques sont souvent trouvées dans les formations arbustives qui marquent la limite entre la forêt et les champs qui abritent des sentiers de randonnée ou des sentiers empruntés par les animaux (16). En effet, elles peuvent proliférer dans de petits bosquets, y compris ceux que l'on peut retrouver dans les cours arrière résidentielles, mais sont rarement détectées dans les pelouses, en particulier celles qui sont gardées courtes $(17,18,19)$. Par conséquent, si les habitats boisés et à l'orée des forêts peuvent être évités, le risque de morsures de tiques est généralement très faible (20). À l'inverse, le fait de visiter de tels habitats accroît l'exposition et devrait provoquer la prise en compte du recours à d'autres mesures de protection, telles que des insectifuges (section 2 ci-dessous).

Figure 1 : Habitat abritant potentiellement des populations de tiques à pattes noires et où elles pourraient être rencontrées

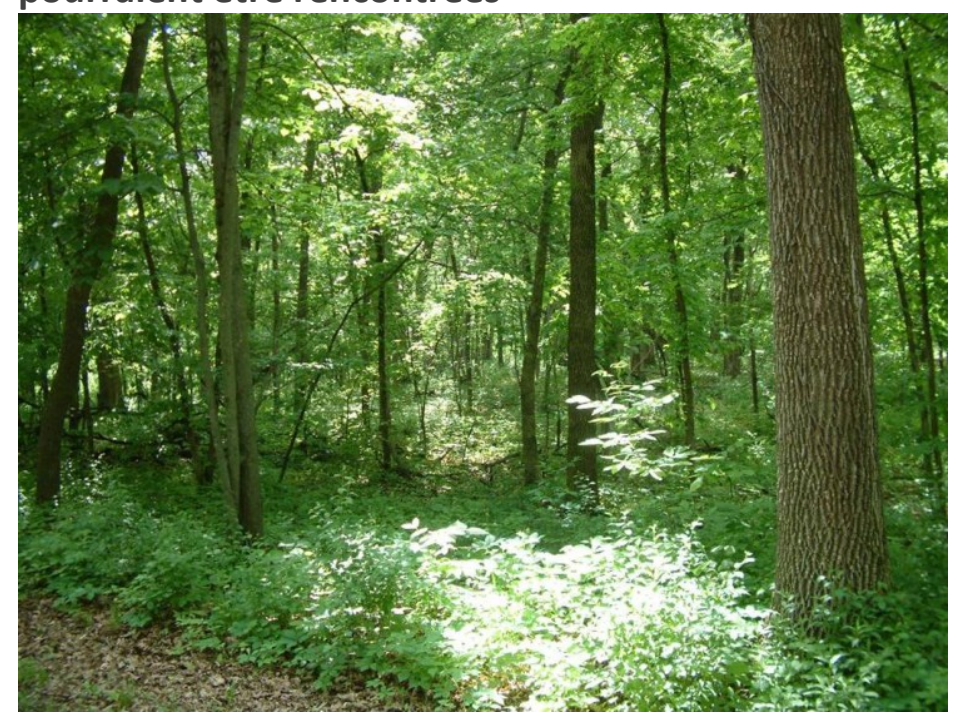

Les tiques sont associées à des régions géographiques et le risque de morsures de tiques et de maladie de Lyme est le plus élevé dans les régions où des populations de tiques sont établies et autonomes. Les morsures de tiques et la maladie de Lyme peuvent cependant survenir dans une zone inconnue pour abriter des populations établies de tiques, soit parce que la surveillance en est encore à déterminer la population ou en raison du petit nombre de tiques " adventives " (populations établies de tiques propagées par les oiseaux migrateurs) présentes $(2,3,13,21)$. Par conséquent, le fait d'éviter les régions où il est connu que des populations de tiques sont établies réduira, mais n'éliminera pas complètement le risque.

Le risque de contracter la maladie de Lyme varie également selon le stade de développement de la tique et selon la saison. Les tiques au stade larvaire (stade le plus précoce) peuvent parfois mordre les gens, mais, puisqu'elles ne sont pas infectées par la BB (22), elles ne représentent pas un risque de maladie de Lyme, même si elles peuvent parfois être infectées par d'autres agents pathogènes. Au Canada, le risque est plus élevé au printemps et en été (de mai à août) lorsque les nymphes (le stade juvénile des tiques qui se sont développées par un régime de larves avant l'âge adulte) sont actives $(23,24)$. Le risque accru associé aux nymphes représente probablement leur abondance relativement plus élevée (comparativement aux adultes), de même que notre efficacité réduite à la trouver et à l'éliminer à ce stade de développement avant que la transmission ait lieu (25). II existe également des risques plus tôt au printemps ainsi qu'à l'automne lorsque les tiques adultes sont les plus actives (26) et en théorie en hiver si les températures sont supérieures au point de congélation et qu'il n'y a pas de couvert neigeux sur le terrain $(16,27)$. Utiliser des mesures de protection individuelle ou éviter les régions à 
risque au cours des périodes de l'année où les nymphes et les tiques adultes sont très actives réduira ou éliminera le risque d'exposition (28).

\section{Prévention de la maladie de Lyme par des vêtements appropriés et par le retrait des tiques} Les personnes qui travaillent à l'extérieur ou se livrent à des activités de plein air comme le golf, la chasse, le camping, la pêche et la randonnée pourraient ne pas être en mesure d'éviter les habitats des tiques, mais elles peuvent réduire leur risque de contracter la maladie de Lyme. Le fait de se vêtir adéquatement et de retirer la tique fixée dès que possible constitue des mesures de prévention.

Les tiques à pattes noires attendent généralement sur les feuilles mortes au sol, ou sur les arbrisseaux, qu'un hôte passe. À partir de ce support, elles s'agrippent à un hôte qui passe, puis cherchent à trouver un endroit où se nourrir $(16,29)$. Le fait de porter des vêtements appropriés comme des chaussures fermées, une chemise longue enserrée dans le pantalon et des chaussettes relevées par-dessus le pantalon limite l'accès à la peau, et protège contre les morsures (30). En outre, le fait que la tique ne puisse pas pénétrer dans les vêtements la force à voyager plus longtemps à la surface des vêtements, ce qui accroît la probabilité qu'elle soit découverte avant qu'elle ne puisse mordre pour se nourrir. Le fait de porter des vêtements de couleur pâle favorise la découverte des tiques (31). Pour tuer les tiques restant sur les vêtements après utilisation, mettre les vêtements dans la sécheuse au cycle le plus chaud, puis les laver et les sécher de nouveau (32).

Les tiques vectrices se nourrissent sur leurs hôtes humains pour une période allant jusqu'à sept jours (16). II a été bien établi chez les modèles animaux que la plupart des cas de transmission de la BB ne se produisaient pas avant une période d'un jour ou plus après que les tiques aient commencé à se nourrir $(33,34,35)$. Par conséquent, le retrait des tiques dans un délai de 24 à 36 heures devrait prévenir l'infection par $B$. burgdorferi dans la plupart des cas. En effet, il a été démontré qu'une inspection quotidienne de la peau pour rechercher des tiques, et le bain ou la douche dans une période de quelques heures suivant les activités extérieures (ce qui augmente les probabilités de trouver des tiques), réduisent le risque de contracter la maladie de Lyme (36). Habituellement, une vérification de la présence de tiques devrait être effectuée en quittant une zone à risque, même s'il est prudent de vérifier la présence de tiques en mouvement sur les vêtements ou la peau alors que l'on se trouve dans la zone à risque.

Pour retirer une tique il est préférable d'utiliser des pinces à épiler avec des extrémités rigides et en angle placées près de la tête de la tique et aussi près de la peau que possible, et de tirer en effectuant un mouvement vers le haut $(34,37,38,39)$ comme le montre la Figure 2 . Après que la tique est retirée, la zone piquée doit être lavée avec du savon et de l'eau et traitée à l'aide d'un antiseptique. 
Figure 2 : Diagramme illustrant la méthode privilégiée pour le retrait de la tique fixée sur la peau

Extraire une tique avec des pinces :

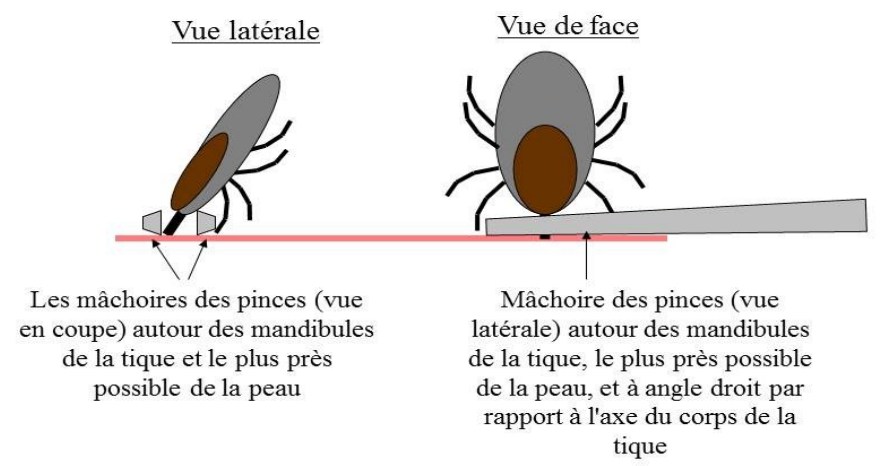

\section{Prévention de la maladie de Lyme par des barrières chimiques}

Les insectifuges topiques peuvent prévenir les piqûres d'un large éventail d'insectes vecteurs, y compris les tiques $(40,41)$. Au Canada, l'insectifuge le plus largement accessible est le N,N-diéthyl-m-toluamide (DEET). II protège contre les morsures de tiques, son innocuité a été examinée par Santé Canada et il constitue un ingrédient actif privilégié pour une protection contre un éventail d'autres agents pathogènes transmis par les insectes $(40,42,43)$. En général, les produits qui contiennent de fortes concentrations de DEET (p. ex., de 20 à $30 \%$ ) procurent une protection de plus longue durée (44).

Ces produits à plus fortes concentrations sont homologués pour utilisation chez les adultes et les enfants âgés de plus de 12 ans. Chez les enfants âgés de 2 à 12 ans, on peut utiliser les produits qui contiennent jusqu'à $10 \%$ de DEET, mais pas plus de trois fois par jour. Pour les enfants âgés de 6 à 24 mois, des concentrations de DEET allant jusqu'à $10 \%$ peuvent être utilisées, mais qu'une seule fois par jour $(44,45)$.

Depuis peu, les insectifuges contenant un ingrédient appelé icaridine, qui fournit des niveaux et des périodes de protection semblables au DEET, sont commercialisés au Canada (46). L'icaridine (aussi connue sous les noms Picaridine et KBR 3023), utilisée dans d'autres pays depuis un certain temps, est recommandée pour se protéger contre les piqûres de tiques $(40,43)$ et, contrairement au DEET, son utilisation à de fortes concentrations (p. ex., $20 \%$ ) n'est pas assujettie à des limites d'âge (http://publications.gc.ca/collections/collection-2011/schc/H113-9-2011-10-fra.pdf). Par conséquent, si une protection pendant de plus longues périodes est nécessaire pour des enfants, l'icaridine pourrait constituer le meilleur insectifuge. Au moment de la rédaction du présent rapport, les produits contenant de l'icaridine n'étaient pas largement accessibles dans les commerces de détail au Canada. Toutefois, plusieurs entreprises prévoient commercialiser des produits (y compris ceux qui contiennent $20 \%$ d'icaridine) à l'échelle du pays dans un proche avenir (c.-à-d. au printemps ou à l'été 2015). Pour tous les insectifuges topiques, il est important de lire et de suivre toutes les directives de l'étiquette.

D'autres insecticides tels que la perméthrine, lorsqu'elle est imprégnée dans les vêtements, agissent aussi comme une mesure de protection personnelle contre les piqûres de tiques $(47,48)$. Une récente étude par essai contrôlé randomisé (ECR) chez des personnes à risque élevé d'exposition à des tiques a démontré qu'elle offre une protection importante (>90\%) par rapport aux sujets utilisant des mesures de protection conventionnels contre les morsures de tiques (49). 
La perméthrine n'est actuellement pas offerte au grand public au Canada, mais il est recommandé que les Canadiens qui voyagent dans des régions des États-Unis et ailleurs (p. ex. en Europe) où la maladie est endémique (40) appliquent de la perméthrine sur leurs vêtements ou utilisent des vêtements prétraités à la perméthrine. On peut se procurer ces produits dans certaines cliniques de santé-voyage ou dans des magasins de plein air lorsqu'on voyage aux États-Unis (40).

Les produits qui contiennent la perméthrine ou d'autres ingrédients actifs qui ne sont pas spécialement approuvés pour le traitement des vêtements visant à prévenir les morsures de tiques ne doivent pas être utilisés à cet effet, car ils n'ont pas été conçus pour une telle utilisation. Ils peuvent être inefficaces et présenter un risque.

\section{Prévention de la maladie par la réduction du nombre de tiques dans l'environnement}

Les approches de gestion et d'aménagement du terrain ou d'application de pesticides ont démontré un certain succès dans la réduction des contacts entre les tiques et les humains aux États-Unis et peuvent jouer un rôle au Canada.

Des modifications simples comme l'émondage des arbres et des arbustes peuvent rendre une zone moins propice à accueillir des tiques. Des zones inhospitalières pour les tiques peuvent aussi être créées autour des cours et des aires de loisirs par l'intégration de structures paysagères (p. ex., une terrasse surélevée) et l'adoption de mesures de base ( $p$. ex. la tonte des pelouses et le nettoyage des broussailles) (50). Pour les personnes vivant dans des régions où les tiques sont présentes, des clôtures (de deux à trois mètres de hauteur) pour éloigner les chevreuils des propriétés réduisent le risque de contracter la maladie de Lyme (36).

L'application de pesticides sur la végétation dans les endroits où l'on trouve des tiques, comme c'est le cas dans les zones de transition entre les bosquets et les pelouses, peut considérablement réduire les populations de tiques $(9,51,52)$. Cette approche n'a pas été largement adoptée au Canada, peut-être parce que la maladie de Lyme constitue un problème " nouveau » et que le marché n'a pas encore été développé. Cette situation pourrait aussi refléter une préoccupation à l'égard du coût et de l'innocuité des pesticides (53). À l'avenir, le public, les organisations et les municipalités devront trouver un juste équilibre entre l'utilisation des pesticides pour contrôler les tiques d'une part, et les coûts, les avantages et la législation actuelle et future liés à l'application des pesticides d'autre part.

Le traitement des cerfs (l'hôte principal de la tique adulte) et des rongeurs (le principal réservoir de l'agent pathogène qui transmet la maladie de Lyme) avec des acaricides pour tuer les tiques a démontré son efficacité par des études de démonstration de principe $(54,55)$. Toutefois, il existe peu de données démontrant que l'abattage de cerfs est efficace pour réduire l'abondance des tiques, sauf dans les environnements particuliers comme c'est le cas des zones insulaires (56).

\section{Conclusion}

Au cours des dernières années, la maladie de Lyme au Canada a évolué d'un problème inhabituel et circonscrit, à un enjeu émergent et en progression. De plus en plus, les populations de tiques et, par conséquent, le risque de contracter la maladie de Lyme empiètent sur les zones densément peuplées. Cette tendance devrait se poursuivre, et exposer la population canadienne à un risque accru d'exposition à des morsures de tiques et à la maladie de Lyme. Mise à part la modification du milieu afin de réduire les facteurs de risque environnementaux, les outils de prévention des piqûres et de l'infection à la BB, sont grandement limités aux approches prônant l'utilisation d'insectifuges et la vérification de la présence de tiques. Bien que des données probantes démontrent l'efficacité de ces interventions (bien que l'évaluation de la mesure de l'effet et de la qualité des preuves n'aient pas été soumises à des examens systématiques), leur efficacité est limitée par un faible taux d'observance (57, $58,59)$. Des efforts visant à informer les Canadiens du risque de contracter la maladie de Lyme et visant à les encourager à se protéger contre les piqûres de moustiques et la maladie sont justifiés. Toutefois, de nouveaux outils et approches sont également nécessaires, en particulier lorsqu'ils viennent compléter les stratégies existantes. Cela peut comprendre des approches novatrices qui encouragent l'adoption de méthodes existantes 
et qui accroissent l'observance des méthodes de protection personnelles par le public; une adoption plus large de méthodes existantes et novatrices de contrôle des populations de tiques (ou de la BB chez les tiques et les animaux réservoirs); la mise au point et l'utilisation de vaccins efficaces pour les humains et acceptés par le public; et l'amélioration continue d'outils d'évaluation et de prévision des risques.

\section{Résumé des recommandations}

\begin{tabular}{|c|c|c|}
\hline Stratégie/Méthode & Raison de l'utilisation & $\begin{array}{l}\text { Références } \\
\text { choisies }\end{array}$ \\
\hline $\begin{array}{l}\text { Utiliser des insectifuges topiques approuvés - } \\
\text { DEET ou icaridine - (suivre les directives de } \\
\text { l'étiquette) et porter des vêtements imprégnés } \\
\text { d'insectifuge (où cela est autorisé). }\end{array}$ & $\begin{array}{l}\text { Prévenir les piqûres de tiques; certains produits } \\
\text { peuvent tuer les tiques. }\end{array}$ & $(40,42,60)$ \\
\hline $\begin{array}{l}\text { Vérifier la présence de tiques au moins une fois par } \\
\text { jour et retirer les tiques découvertes. }\end{array}$ & $\begin{array}{l}\text { Retirer la tique avant que la transmission de } \\
\text { l'agent pathogène de la maladie de Lyme se } \\
\text { produise. }\end{array}$ & $(38,61)$ \\
\hline Éviter les habitats infestés de tiques. & Prévenir l'exposition aux tiques. & $(10,62)$ \\
\hline $\begin{array}{l}\text { Prendre un bain ou une douche dans les deux } \\
\text { heures suivant le départ de l'habitat des tiques. }\end{array}$ & $\begin{array}{l}\text { Déloger les tiques et fournir une autre occasion } \\
\text { de retirer les tiques fixées sur la peau. }\end{array}$ & (36) \\
\hline $\begin{array}{l}\text { Porter des vêtements appropriés (p. ex. chemises } \\
\text { longues et de couleur claire, chaussettes et } \\
\text { pantalons longs). }\end{array}$ & $\begin{array}{l}\text { Limités ou retarder l'accès par les tiques aux } \\
\text { sites de fixation sur la peau et améliorer la } \\
\text { capacité à détecter (et à retirer) les tiques sur } \\
\text { les vêtements. }\end{array}$ & (30) \\
\hline $\begin{array}{l}\text { Modifier l'aménagement des terrains pour réduire le } \\
\text { risque de morsures par les tiques : installer des } \\
\text { clôtures (de plus de } 2 \text { mètres de hauteur), émonder } \\
\text { les arbres et les arbustes des aires de jeu, créer } \\
\text { des zones inhospitalières pour les tiques en bordure } \\
\text { des aires de jeu. }\end{array}$ & $\begin{array}{l}\text { Réduire l'entrée des hôtes des tiques sur les } \\
\text { propriétés et réduire le nombre de tiques dans } \\
\text { les aires de jeu en compromettant leur survie. }\end{array}$ & $(36,50)$ \\
\hline
\end{tabular}

\section{Remerciements}

Les auteurs tiennent à remercier le Comité consultatif de la lutte antiparasitaire du ministère de la Défense nationale pour avoir fourni une évaluation inédite sur l'efficacité de l'icaridine comme insectifuge contre les tiques (et les moustiques).

\section{Conflit d'intérêts}

Aucun

\section{Références}

(1) Ogden NH, Lindsay LR, Morshed M, Sockett PN, Artsob H. The emergence of Lyme disease in Canada. CMAJ. 2009;180(12):12214.

(2) Ogden $\mathrm{NH}$, Koffi JK, Pelcat $\mathrm{Y}$, Lindsay LR. Environmental risk from Lyme disease in central and eastern Canada: a summary of recent surveillance information. Can Commun Dis Rep. 2014;40-5:74-82.

Disponible en français: http://www.phac-aspc.gc.ca/publicat/ccdr-rmtc/14vol40/dr-rm40-05/dr-rm40-05-1-fra.php.

(3) Ogden NH, Koffi JK, Lindsay LR. Assessment of a screening test to identify Lyme disease risk. Can Commun Dis Rep. 2014;405:83-7.

(4) Ogden NH, Lindsay LR, Leighton PA. Predicting the rate of invasion of the agent of Lyme disease Borrelia burgdorferi. J Appl Ecol. 2013;50(2):510-8.

(5) Leighton PA, Koffi JK, Pelcat Y, Lindsay LR, Ogden NH. Predicting the speed of tick invasion: An empirical model of range expansion for the Lyme disease vector Ixodes scapularis in Canada. J Appl Ecol. 2012;49(2):457-64.

(6) Poland GA. Vaccines against Lyme disease: What happened and what lessons can we learn? Clin Infect Dis. 2011 February 01;52(suppl 3):s253-8.

(7) Poland GA. Prevention of Lyme disease: A review of the evidence. Mayo Clin Proc. 2001 Jul;76(7):713-24. 
(8) Corapi KM, White MI, Phillips CB, Daltroy LH, Shadick NA, Liang MH. Strategies for primary and secondary prevention of Lyme disease. Nat Clin Pract Rheumatol. 2007 Jan;3(1):20-5.

(9) Hayes EB, Maupin GO, Mount GA, Piesman J. Assessing the prevention effectiveness of local Lyme disease control. J Public Health Manag Pract. 1999;5(3):84-92.

(10) Piesman J, Eisen L. Prevention of tick-borne diseases. Annu Rev Entomol. 2008;53:323-43.

(11) Piesman J, Beard CB. Prevention of tick-borne diseases. J Environ Health. 2012;74(10):30-2

(12) Ogden NH, Lindsay LR, Morshed M. The emergence of Lyme disease in Canada. CMAJ. 2009;181(5):291.

(13) Ogden NH, Trudel L, Artsob H, Barker IK, Beauchamp G, Charron DF, et al. Ixodes scapularis ticks collected by passive surveillance in Canada: Analysis of geographic distribution and infection with Lyme borreliosis agent Borrelia burgdorferi. J Med Entomol. 2006;43(3):600-9.

(14) Guerra M, Walker E, Jones C, Paskewitz S, Cortinas MR, Stancil A, et al. Predicting the risk of Lyme disease: Habitat suitability for Ixodes scapularis in the north central United States. Emerg Infect Dis. 2002 Mar;8(3):289-97.

(15) Lindsay LR, Mathison SW, Barker IK, McEwen SA, Surgeoner GA. Abundance of Ixodes scapularis (Acari: Ixodidae) larvae and nymphs in relation to host density and habitat on Long Point, Ontario. J Med Entomol. 1999;36(3):243-54.

(16) Smith RP. Ticks: The vectors of Lyme disease. In: Halperin JJ, editor. Lyme disease: An evidence-based approach. Wallingford, Oxfordshire, UK: CABI; 2011. p. 1-28.

(17) Duffy DC, Clark DD, Campbell SR, Gurney S, Perello R, Simon N. Landscape patterns of abundance of Ixodes scapularis (Acari: Ixodidae) on Shelter Island, New York. J Med Entomol. 1994 Nov;31(6):875-9.

(18) Carroll MC, Ginsberg HS, Hyland KE, Hu R. Distribution of Ixodes dammini (Acari: Ixodidae) in residential lawns on Prudence Island, Rhode Island. J Med Entomol. 1992 Nov;29(6):1052-5.

(19) Maupin GO, Fish D, Zultowsky J, Campos EG, Piesman J. Landscape ecology of Lyme disease in a residential area of Westchester County, New York. Am J Epidemiol. 1991;133(11):1105-13.

(20) Piesman J, Humair P. The spirochetes and vector ticks of Lyme borreliosis in nature. In: Lyme Borreliosis in Europe and North American: Epidemiology and clinical practice. Hoboken, N.J.: John Wiley \& Sons, Inc.; 2011. p. 37-51.

(21) Diuk-Wasser MA, Hoen AG, Cislo P, Brinkerhoff R, Hamer SA, Rowland M, et al. Human risk of infection with Borrelia burgdorferi, the Lyme disease agent, in eastern United States. Am J Trop Med Hyg. 2012 Feb;86(2):320-7.

(22) Rollend L, Fish D, Childs JE. Transovarial transmission of Borrelia spirochetes by Ixodes scapularis: A summary of the literature and recent observations. Ticks Tick Borne Dis. 2013;4(1-2):46-51.

(23) Bacon RM, Kugeler KJ, Mead PS. Centers for Disease Control and Prevention (CDC). Surveillance for Lyme disease--United States, 1992-2006. MMWR Surveill Summ. 2008 Oct 3;57(10):1-9.

(24) Rodgers SE, Miller NJ, Mather TN. Seasonal variation in nymphal blacklegged tick abundance in southern New England forests. J Med Entomol. 2007 Sep;44(5):898-900.

(25) Falco RC, Fish D, Piesman J. Duration of tick bites in a Lyme disease-endemic area. Am J Epidemiol. 1996 Jan 15;143(2):187-92.

(26) Lindsay LR, Mathison SW, Barker IK, McEwen SA, Gillespie TJ, Surgeoner GA. Microclimate and habitat in relation to Ixodes scapularis (Acari: Ixodidae) populations on Long Point, Ontario, Canada. J Med Entomol. 1999;36(3):255-62.

(27) Duffy DC, Campbell SR. Ambient air temperature as a predictor of activity of adult Ixodes scapularis (Acari: Ixodidae). J Med Entomol. 1994 Jan;31(1):178-80.

(28) Clark RP, Hu LT. Prevention of Lyme disease and other tick-borne infections. Infect Dis Clin North Am. 2008;22(3):381-96.

(29) Williams SC, Ward JS. Effects of Japanese barberry (Ranunculales: Berberidaceae) removal and resulting microclimatic changes on Ixodes scapularis (Acari: Ixodidae) abundances in Connecticut, USA. Environ Entomol. 2010 Dec;39(6):1911-21.

(30) Carroll JF, Kramer M. Different activities and footwear influence exposure to host-seeking nymphs of Ixodes scapularis and Amblyomma americanum (Acari: Ixodidae). J Med Entomol. 2001 Jul;38(4):596-600.

(31) Stjernberg L, Berglund J. Detecting ticks on light versus dark clothing. Scand J Infect Dis. 2005;37(5):361-4.

(32) Carroll JF. A cautionary note: Survival of nymphs of two species of ticks (Acari: Ixodidae) among clothes laundered in an automatic washer. J Med Entomol. 2003 Sep;40(5):732-6.

(33) Hojgaard A, Eisen RJ, Piesman J. Transmission dynamics of Borrelia burgdorferi s.s. during the key third day of feeding by nymphal Ixodes scapularis (Acari: Ixodidae). J Med Entomol. 2008 Jul;45(4):732-6.

(34) Piesman J, Dolan MC. Protection against Lyme disease spirochete transmission provided by prompt removal of nymphal Ixodes scapularis (Acari: Ixodidae). J Med Entomol. 2002 May;39(3):509-12.

(35) Piesman J, Mather TN, Sinsky RJ, Spielman A. Duration of tick attachment and Borrelia burgdorferi transmission. J Clin Microbiol. 1987 Mar;25(3):557-8.

(36) Connally NP, Durante AJ, Yousey-Hindes KM, Meek JI, Nelson RS, Heimer R. Peridomestic Lyme disease prevention. Results of a population-based case-control study. Am J Prev Med. 2009;37(3):201-6.

(37) Needham GR. Evaluation of five popular methods for tick removal. Pediatrics. 1985 Jun;75(6):997-1002.

(38) Prevention of Lyme borreliosis. In: Sood SK, editor. Lyme borreliosis in Europe and North America: Epidemiology and clinical practice. Hoboken, N.J.: John Wiley \& Sons, Inc.; 2011. p. 225-44.

(39) Duscher GG, Peschke R, Tichy A. Mechanical tools for the removal of Ixodes ricinus female ticks: Differences of instruments and pulling or twisting? Parasitol Res. 2012 Oct;111(4):1505-11.

(40) Schofield S, Plourde P. Statement on personal protective measures to prevent arthropod bites . An Advisory Committee Statement (ACS). Can Commun Dis Rep. 2012 Nov 2012;38(ACS-3):1-18.

(41) Vázquez M, Muehlenbein C, Cartter M, Hayes EB, Ertel S, Shapiro ED. Effectiveness of personal protective measures to prevent Lyme disease. Emerg Infect Dis. 2008;14(2):210-6.

(42) Katz TM, Miller JH, Hebert AA. Insect repellents: Historical perspectives and new developments. J Am Acad Dermatol. 2008 May;58(5):865-71.

(43) Centre for Disease Control [Internet]. Preventing tick bites. Atlanta, GA: Centers for Disease Control and Prevention; 2015. http://www.cdc.gov/lyme/prev/index.html. 
(44) Health Canada. ARCHIVED - Memo - DEET (N,N-diethyl-m-toluamide) Statement of Guarantee and Labelling Allowances. PMRA; 2010. http://www.hc-sc.gc.ca/cps-spc/pest/registrant-titulaire/prod/_memo-note/deet-eng.php.

Disponible en français: http://www.hc-sc.gc.ca/cps-spc/pest/registrant-titulaire/prod/_memo-note/deet-fra.php.

(45) Health Canada Insect Repellents. PMRA; 2014 http://www.healthycanadians.gc.ca/environmentenvironnement/pesticides/insect_repellents-insectifuges-eng.php.

Disponible en français: http://www.canadiensensante.gc.ca/healthy-living-vie-saine/environment-

environnement/pesticides/insect_repellents-insectifuges-fra.php?_ga=1.62978415.1575686084.1412886377.

(46) Health Canada. Registration Decision RD2012-05, Icaridin.PMRA; 2012. http://www.hc-sc.gc.ca/cpsspc/pubs/pest/_decisions/rd2012-05/index-eng.php.

Disponible en français: http://www.hc-sc.gc.ca/cps-spc/pubs/pest/_decisions/rd2012-05/index-fra.php.

(47) Miller NJ, Rainone EE, Dyer MC, González ML, Mather TN. Tick bite protection with permethrin-treated summer-weight clothing. J Med Entomol. 2011;48(2):327-33.

(48) Evans SR, Korch GW, Lawson MA. Comparative field evaluation of permethrin and deet-treated military uniforms for personal protection against ticks (Acari). J Med Entomol. 1990 Sep;27(5):829-34.

(49) Vaughn MF, Meshnick SR. Pilot study assessing the effectiveness of long-lasting permethrin-impregnated clothing for the prevention of tick bites. Vector Borne Zoonotic Dis. 2011 Jul;11(7):869-75.

(50) Stafford KC. Tick management handbook: An integrated guide for homeowners, pest control operators and public health officials for the prevention of tick-associated disease. New Haven, Conn.: Connecticut Agricultural Experiment Station; 2007.

(51) Schulze TL, Jordan RA, Hung RW, Taylor RC, Markowski D, Chomsky MS. Efficacy of granular deltamethrin against Ixodes scapularis and Amblyomma americanum (Acari: Ixodidade) nymphs. J Med Entomol. 2001 Mar;38(2):344-6.

(52) Schulze TL, Jordan RA, Krivenko AJ. Effects of barrier application of granular deltamethrin on subadult Ixodes scapularis (Acari: Ixodidae) and nontarget forest floor arthropods. J Econ Entomol. 2005 Jun;98(3):976-81.

(53) Gould LH, Nelson RS, Griffith KS, Hayes EB, Piesman J, Mead PS, et al. Knowledge, attitudes and behaviors regarding Lyme disease prevention among Connecticut residents, 1999-2004. Vector Borne Zoonotic Dis. 2008;8(6):769-76.

(54) Dolan MC, Maupin GO, Schneider BS, Denatale C, Hamon N, Cole C, et al. Control of immature Ixodes scapularis (Acari: Ixodidae) on rodent reservoirs of Borrelia burgdorferi in a residential community of southeastern Connecticut. J Med Entomol. 2004 Nov;41(6):1043-54.

(55) Brei B, Brownstein JS, George JE, Pound JM, Miller JA, Daniels TJ, et al. Evaluation of the United States Department Of Agriculture Northeast Area-wide Tick Control Project by meta-analysis. Vector Borne Zoonotic Dis. 2009 Aug;9(4):423-30.

(56) Rand PW, Lubelczyk C, Holman MS, Lacombe EH, Smith RP. Abundance of Ixodes scapularis (Acari: Ixodidae) after the complete removal of deer from an isolated offshore island, endemic for Lyme Disease. J Med Entomol. 2004 Jul;41(4):779-84.

(57) Bayles BR, Evans G, Allan BF. Knowledge and prevention of tick-borne diseases vary across an urban-to-rural human land-use gradient. Ticks Tick Borne Dis. 2013 Jun;4(4):352-8.

(58) Schofield S, Crane F, Tepper M. Good interventions that few use: Uptake of insect bite precautions in a group of Canadian Forces personnel deployed to Kabul, Afghanistan. Mil Med. 2012;177(2):209-15.

(59) Herrington JE. Risk perceptions regarding ticks and Lyme disease: A national survey. Am J Prev Med. 2004;26(2):135-40.

(60) Bissinger BW, Roe RM. Tick repellents: Past, present, and future. Pestic Biochem Physiol. 2010;96(2):63-79.

(61) Shadick NA, Daltroy LH, Phillips CB, Liang US, Liang MH. Determinants of tick-avoidance behaviors in an endemic area for Lyme disease. Am J Prev Med. 1997 Jul-Aug;13(4):265-70.

(62) Eisen RJ, Piesman J, Zielinski-Gutierrez E, Eisen L. What do we need to know about disease ecology to prevent Lyme disease in the northeastern United States? J Med Entomol. 2012;49(1):11-22. 